\title{
Fringe field effects in small rings of large acceptance
}

\author{
Martin Berz, Béla Erdélyi, and Kyoko Makino \\ Department of Physics and Astronomy and National Superconducting Cyclotron Laboratory, Michigan State University, \\ East Lansing, Michigan 48824
}

(Received 3 August 2000; published 6 December 2000)

\begin{abstract}
Recently there has been renewed interest in the influence of fringe fields on particle dynamics, due to studies that revealed their importance in some cases, as, for example, the proposed Neutrino Factory and muon colliders. In this paper, we present a systematic study of generic fringe field effects. Using as an example a lattice of the proposed Neutrino Factory, we show that fringe fields influence the dynamics of particles at all orders, starting with the linear motion. It is found that the widely used sharp cutoff approximation leads to divergences regardless of the specific fall-off shape of the fields. The results suggest that a careful consideration of fringe field effects in the design stage of small machines for large emittances is always recommended.
\end{abstract}

PACS numbers: 41.75.-i, 29.27.-a, 41.85.Ja

\section{INTRODUCTION}

Because of the diversity of the field of nonlinear beam optics, the mathematical methods employed and the formalisms utilized can be very different depending on the specific design requirements. One of the topics for which traditionally very different approaches have been carried out in different subfields is the fringe fields, or end fields. For the purpose of simulations of large storage rings, fringe field effects are often neglected. Sometimes this is a quite good approximation. However, strictly speaking, it is an unphysical model, as the electromagnetic fields of the model do not satisfy Maxwell's equations. The simplest method to take fringe fields into account is to approximate their effect with a kick characterized by the integrated field value [1]. While this model may alleviate some problems, it is not a cure, and more sophisticated models are needed for accurate simulations. Besides the kick, the effect of the fringe fields has been characterized by a sudden shift in position at the so-called effective field boundary [2-5]. For a specific field falloff, in [6] the third-order aberrations and their scaling with fringe field extension have been computed for the quadrupole. Also, leading order hard-edge fringe field effects have been studied in [7]. Fringe fields have been shown to adversely affect the PEP-II dynamic aperture [8]. However, an extensive study of fringe field effects in small rings has so far not been available.

The nonlinearities due to fringe fields have been well known in the field of high resolution particle spectrographs for a long time [4,9]. Also, as has been shown recently, they tend to become significant in small rings, especially at larger emittances $[10,11]$. The latter studies motivated us to look more deeply, at a fundamental level, at the fringe field effects. We keep the study generic because actual lattices for the muon storage rings are available at a preliminary design stage, and to illustrate general trends in small footprint, large acceptance rings.

For a better understanding of the fringe field effects, in this paper we perform a study of the effects that one may miss by not considering the influence of the fringe fields. As an example we use a version of the proposed $30 \mathrm{GeV}$ Neutrino Factory [12]. The fringe fields' falloffs are modeled by a six parameter Enge function where $s$ is the arc length along the reference trajectory, used as the independent variable, $D$ denotes the full aperture of the magnet, and the $a_{i}(i=1,2, \ldots, 6)$ are called Enge coefficients. We look at fringe field effects as a function of magnet aperture and fall-off shape. This is achieved by varying the magnet apertures $D$ and the Enge coefficients $a_{i}$.

The fringe field effects can be particularly easily studied in the map picture using differential algebraic methods [13-15]. The consequences of the fringe field effects influence all orders of the motion, beginning with the linear behavior. Their complete treatment to any order is possible in the code COSY INFINITY [16-20]. To quantify the effects we compute linear tunes, amplitude dependent tune shifts, chromaticities, and resonance strengths, and estimate dynamic apertures.

In the following sections we present some basic theory, and the observations related to aperture and shape dependent effects. We will conclude with a short summary.

\section{THEORY}

In the divergence-free and curl-free regions of the magnets it is possible to derive the magnetic field from a 
magnetic scalar potential that satisfies the Laplace equation. The general solution in cylindrical coordinates with axial coordinate $s$, in the so-called multipole expansion form, is

$$
V_{B}=\sum_{k, l=0}^{\infty}\left[b_{k, l}(s) \sin l \phi+a_{k, l}(s) \cos l \phi\right] r^{k} .
$$

The functions $b_{l, l}(s)$ are called the normal and $a_{l, l}(s)$ the skew multipoles, respectively. The components, according to $l=0,1,2,3, \ldots$, are called the solenoid, dipole, quadrupole, sextupole, etc. components, respectively. In the fringe region of the magnets these are $s$ dependent, which induce the following recursion relations (see, for example, [15]):

$$
\begin{aligned}
& b_{l+2 n, l}(s)=\frac{b_{l, l}^{(2 n)}(s)}{\prod_{\nu=1}^{n}\left[l^{2}-(l+2 \nu)^{2}\right]}, \\
& a_{l+2 n, l}(s)=\frac{a_{l, l}^{(2 n)}(s)}{\prod_{\nu=1}^{n}\left[l^{2}-(l+2 \nu)^{2}\right]},
\end{aligned}
$$

where $b_{l, l}^{(2 n)}(s)$ denotes the $2 n$th derivative of $b_{l, l}(s)$ with respect to $s$. Sometimes they are called pseudomultipoles. The coefficients that cannot be obtained by these relations are zero. Hence, the only free parameters in the general form of the potential are the $s$-dependent multipoles. For practical calculations, these are either fitted to represent measured data or obtained by multipole decomposition of detailed field computations as in [21].

The additional pseudomultipole nonlinearities of the fringe fields couple to higher derivatives of the multipole strengths. In practice this entails that fringe field effects become more and more relevant the more the particles are away from the axis of the elements, which, of course, is directly connected to the emittance of the beam. Also, it is clear that the fall-off shapes, and implicitly the apertures, have an influence on the induced nonlinearities.

\section{APERTURE DEPENDENT EFFECTS ON LINEAR TUNES AND CHROMATICITIES}

In some perturbation theories the linear or first-order effects are not considered. In others, the first-order effect is characterized by a kick [1], or as a sudden change in position and momentum at the so-called effective field boundary [4]. Here, using the $30 \mathrm{GeV}$ Neutrino Factory lattice,

TABLE I. Enge coefficients of the default fringe field falloff used in COSY INFINITY for dipoles, quadrupoles, and sextupoles, respectively.

\begin{tabular}{lrrr}
\hline \hline & Dipole & Quadrupole & \multicolumn{1}{c}{ Sextupole } \\
\hline$a_{1}$ & 0.478959 & 0.296471 & 0.176659 \\
$a_{2}$ & 1.911289 & 4.533219 & 7.153079 \\
$a_{3}$ & -1.185953 & -2.270982 & -3.113116 \\
$a_{4}$ & 1.630554 & 1.068627 & 3.444311 \\
$a_{5}$ & -1.082657 & -0.036391 & -1.976740 \\
$a_{6}$ & 0.318111 & 0.022261 & 0.540068 \\
\hline \hline
\end{tabular}

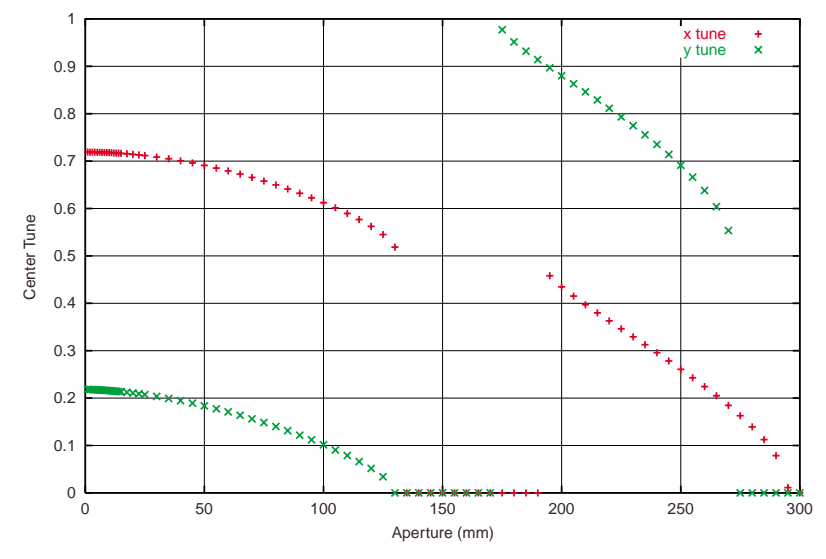

FIG. 1. (Color) Center tunes as a function of aperture. The fringe field shape is given by the default Enge coefficients.

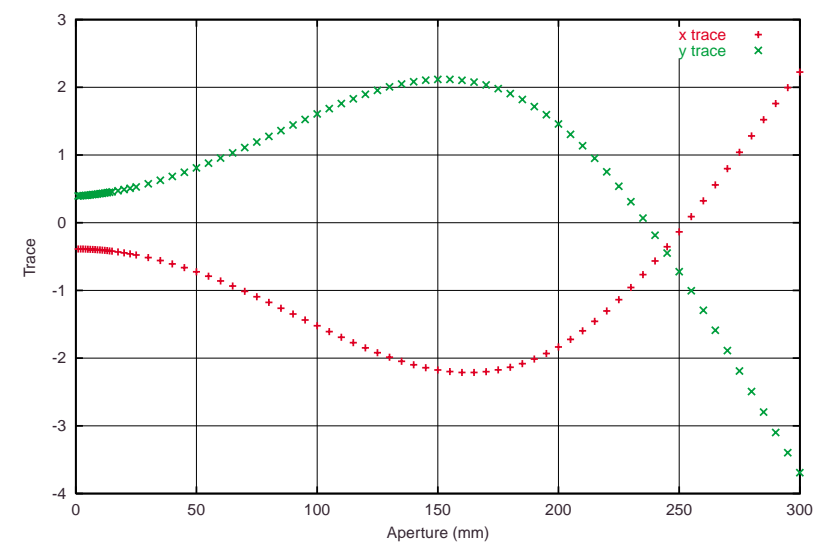

FIG. 2. (Color) Traces of the $x-a$ and $y-b$ submatrices versus aperture. The fringe field shape is given by the default Enge coefficients.

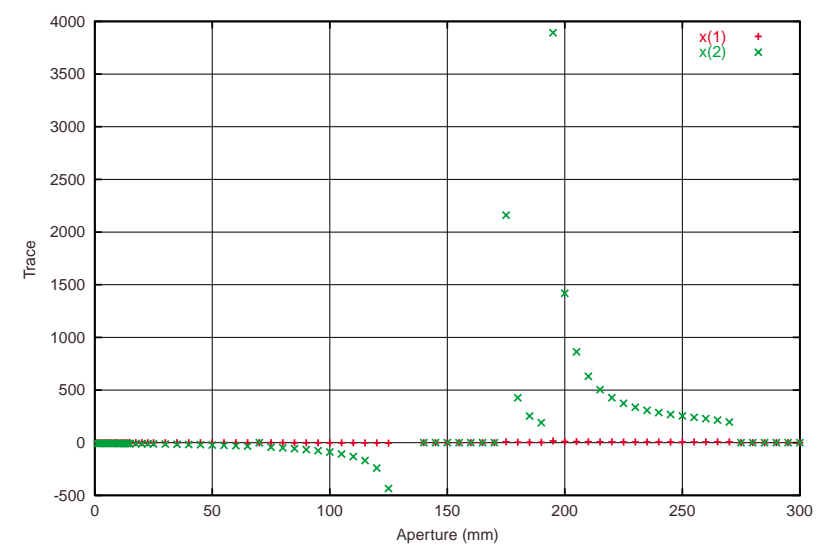

FIG. 3. (Color) First-order and second-order $x$ chromaticities as a function of aperture. The fringe field shape is given by the default Enge coefficients.

we compute with COSY INFINITY the linear tunes and chromaticities as a function of the magnet apertures. We assume that all the magnets have the same aperture, and the falloff is given by the Enge coefficients of Table I [22]. 


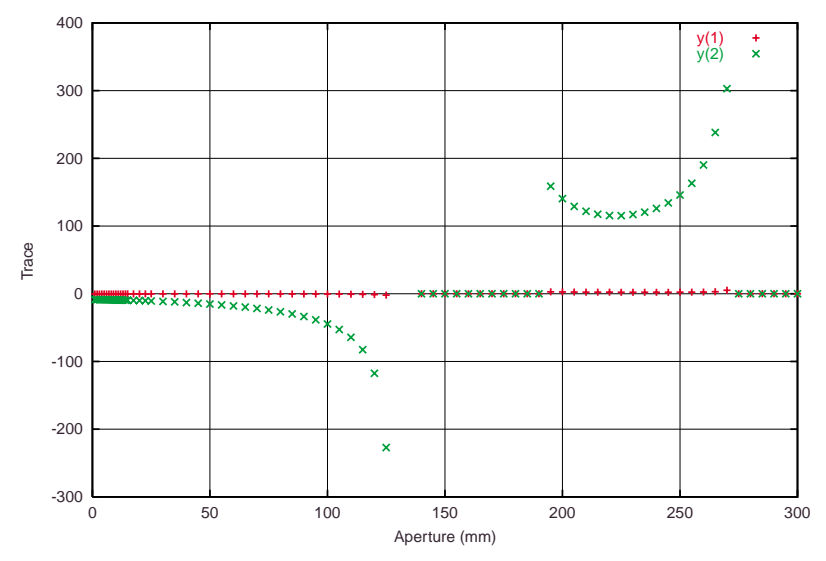

FIG. 4. (Color) First-order and second-order $y$ chromaticities as a function of aperture. The fringe field shape is given by the default Enge coefficients.

We will call them hereafter the default Enge coefficients. It is important to note that the Enge function model can be used for a global fit of the magnetic fields, including the out of axis expansion. This has been demonstrated in several real situations, as for example the National Superconducting Cyclotron Laboratory's S800 spectrograph [23], the GSI quadrupole dipole (QD) kaon spectrometer
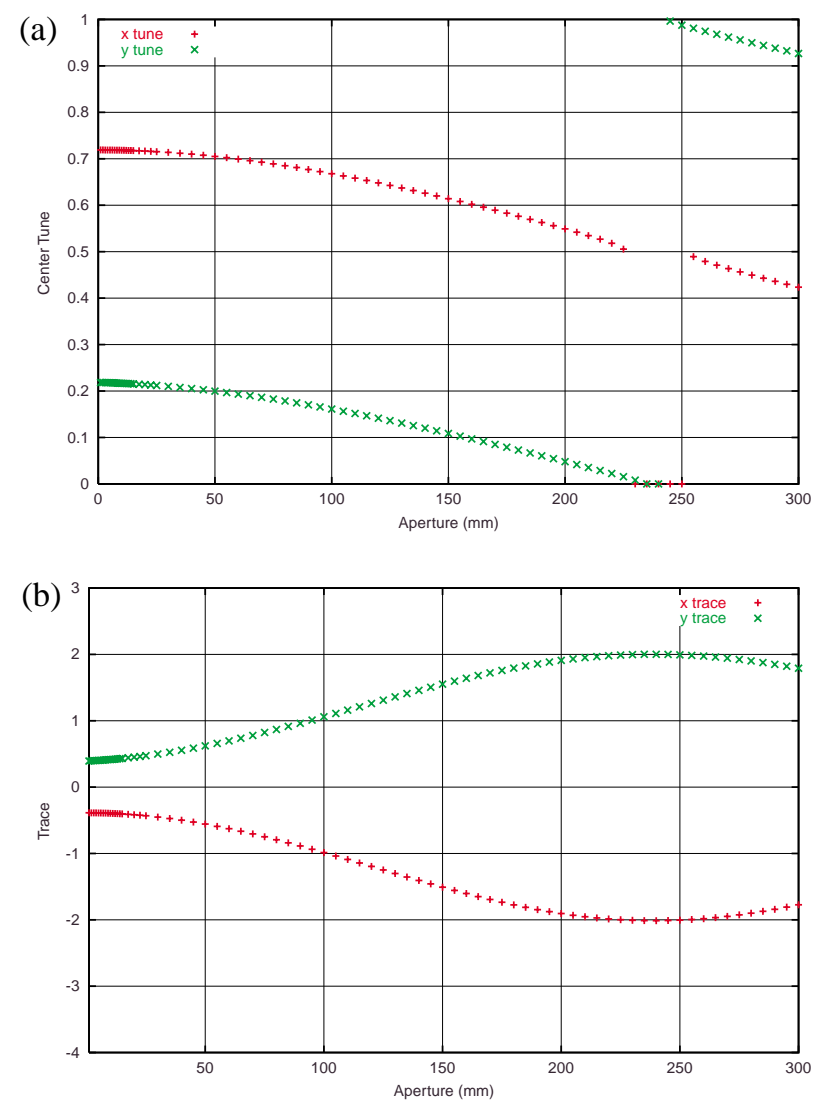

FIG. 5. (Color) (a) Same as Fig. 1 and (b) same as Fig. 2, but without the matching section, where most of the fringe field effects are concentrated.
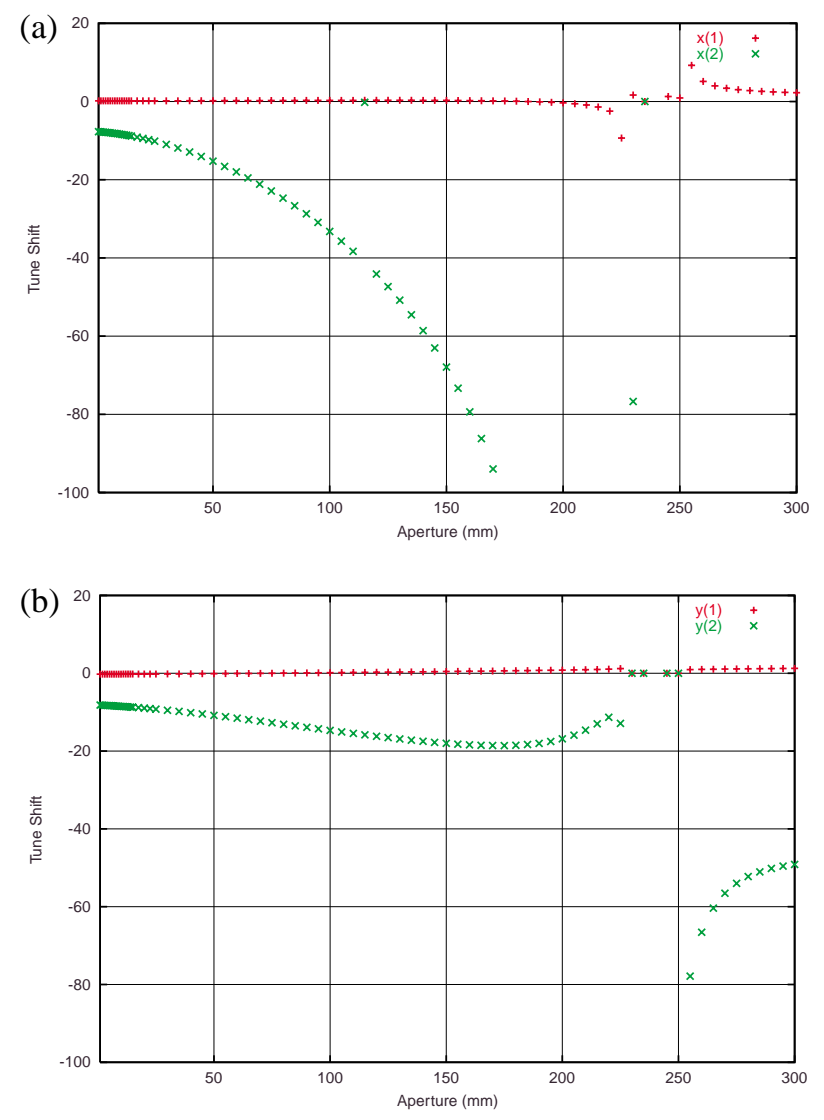

FIG. 6. (Color) (a) Same as Fig. 3 and (b) same as Fig. 4, but without the matching section, where most of the fringe field effects are concentrated.

[24], and even the rather peculiar Large Hadron Collider's high gradient quadrupoles (LHCHGQ) lead end [25].

The aperture is varied between 1 and $300 \mathrm{~mm}$. Figure 1 gives the results for the $x$ and $y$ center tunes. In the stable regions the tunes change continuously and monotonically with the aperture. However, in general there is a nonlinear relationship between center tunes and the aperture. As the linear motion is uncoupled, for linear stability the absolute values of the traces of the $x$-a (horizontal phase plane) and $y-b$ (vertical phase plane) submatrices, respectively, need to be less than 2. The nonlinear dependence of the traces on the apertures is also clear from Fig. 2. The trend regarding chromaticities is included in Figs. 3 and 4.

It has been noticed that the main impact of the fringe fields is coming from only a few matching quadrupoles in the arcs [11]. We repeated the computations of this section for the same ring, with the respective matching quadrupole fringe fields turned off. As one can see from Figs. 5 and 6 , the results are different only quantitatively, but qualitatively the situation remains the same.

\section{THE SHARP CUTOFF LIMIT}

As we already mentioned, the sharp cutoff or hard-edge approximation is a contradiction in itself, as far as the 
physics goes. However, as a purely mathematical approximation it can still be analyzed in some detail. Qualitatively, it can be characterized as follows. The function that describes the falloff is called the cutoff function or a bump function. It is well known in the mathematical literature $[26,27]$ that infinitely often continuously differentiable ( $C^{\infty}$ smooth) bump functions can be found such that they take the value 1 on one closed set, and assume the value 0 on the complement of another closed set; one of the closed sets lying in the interior of the other closed set. For our case the inner closed set can be taken as the region of the main field of the magnet, and the complement of the outer closed set the region where the fields practically vanish. Furthermore, the two sets are arbitrary except for the already mentioned conditions. This means that the two sets can be taken arbitrarily close to each other in some sense (for example in the Hausdorff metric). Thus, the fall-off speed can be arbitrarily fast, and, at the same time, the smoothness can be kept intact.

This is why the sharp cutoff limit gives satisfactory numerical results when fringe fields are not important: in principle, the fall-off region's width always can be taken smaller than the smallest step of any integrator, and thus escapes any numerical issues. On the other hand, as a rigorous mathematical limit, the sharp cutoff limit corresponds to the case where the two closed sets "touch" each other. In this case any bump function tends to the sum of two Heaviside functions, which then end up in the Hamiltonian. However, as is well known, the dynamics are not governed by the Hamiltonian itself, but by its gradient. The Heaviside function's derivative being the Dirac delta function, it follows that the sharp cutoff limit gives rise to divergences. The divergences show up as blowing up of some of the map elements, and, as a consequence, some of the tune shifts and resonance strengths as well.

Hence, blowup of amplitude dependent tune shifts in the sharp cutoff limit occurs regardless of the exact shape of the falloff. However, in the perturbative order-by-order approach the divergence can occur at different orders depending on the specific fields involved. For the case of the homogeneous dipole it has been derived [28] that the lowest order map element that causes divergence is $(b \mid$ yyy); that is the element that shows how the final angle in the vertical phase plane depends on the initial position in the vertical direction. For multipoles with straight optical axes, the divergences occur at higher orders. Recently, under some simplifying assumptions, estimates for the second-order amplitude dependent tune shifts of a quadrupole fringe field have been calculated [29]. It is shown that, in the integrated field approximation, the second-order tune shifts tend to a finite nonzero value in the sharp cutoff approximation. This is derived from a few integrals that are part of the integrated Hamiltonian, and taking limits as the extension of the fringe region goes to zero. Within its domain of validity the estimate gives good agreement with the exact values computed by COSY INFINITY, at least for the cases studied.

The second-order tune shifts are functions of the thirdorder map elements. To compute the third-order map elements of a quadrupole, integrals of the type

$$
I_{2}=\int_{s_{b}}^{s_{e}} r^{3}(s) b_{2,2}^{\prime \prime}(s) d s,
$$

appear, where $b_{2,2}^{\prime \prime}(s)$ is the second-order derivative with respect to $s$ of the quadrupole strength, and $s_{b}$ and $s_{e}$ are the beginning and end of the fringe field, respectively. The function $r(s)$ is the initially unknown orbit. To evaluate the above integral within the framework of perturbation theory, one can distinguish two cases. In the approximation that the orbit does not change over the fringe region, that is $r(s)=$ const over $s \in\left[s_{b}, s_{e}\right]$, the result is $I_{2}=r^{3}\left(s_{b}\right)\left[b_{2,2}^{\prime}\left(s_{e}\right)-b_{2,2}^{\prime}\left(s_{b}\right)\right]$. Because, at the beginning and end, the fringe fields have assumed their plateaus $b(s)=0$ and $b(s)=1$, respectively, we have $b_{2,2}^{\prime}\left(s_{e}\right)=$ $b_{2,2}^{\prime}\left(s_{b}\right)=0$ and, hence, altogether $I_{2}=0$. This approximation yields the prediction that there are no second-order tune shifts, independent of the specific shape of $b_{2,2}(s)$.

On the other hand, we obtain a different and more precise answer performing perturbation theory successively over small equidistant subintervals of the interval $\left[s_{b}, s_{e}\right]$, which, in the limit of all subintervals going to zero, leads to the correct result in much the same way as numerical integration schemes. Again, we assume perturbatively that $r(s)$ is constant over the interval in question, which, in the $i$ th step of the perturbation theory, spans $s \in\left[s_{i}, s_{i+1}\right]$. If $\Delta s=s_{i+1}-s_{i}$ is small enough, this approximation will become better and better. However, unavoidably, $r(s)$ will change slightly over different time steps. Consider the specific case of performing $N$ substeps of the perturbation theory. Then an estimate for $I_{2}$ is

$$
\begin{aligned}
I_{2} \approx & \sum_{i=1}^{N} r^{3}\left(s_{i}\right) \int_{s_{i}}^{s_{i+1}} b_{2,2}^{\prime \prime}(s) d s=\sum_{i=1}^{N} r^{3}\left(s_{i}\right)\left[b_{2,2}^{\prime}\left(s_{i+1}\right)-b_{2,2}^{\prime}\left(s_{i}\right)\right] \\
= & -r^{3}\left(s_{1}\right) b_{2,2}^{\prime}\left(s_{1}\right)-\left[\sum_{i=1}^{N} b_{2,2}^{\prime}\left(s_{i+1}\right)\left[r^{3}\left(s_{i+1}\right)-r^{3}\left(s_{i}\right)\right]\right] \\
& +r^{3}\left(s_{N}\right) b_{2,2}^{\prime}\left(s_{N+1}\right) \\
= & -\sum_{i=1}^{N} b_{2,2}^{\prime}\left(s_{i+1}\right)\left[r^{3}\left(s_{i+1}\right)-r^{3}\left(s_{i}\right)\right]
\end{aligned}
$$


where we used as before that $b_{2,2}^{\prime}\left(s_{1}\right)=b_{2,2}^{\prime}\left(s_{N+1}\right)=0$. Performing a Taylor expansion of $r\left(s_{i+1}\right)$ we have

$$
r^{3}\left(s_{i+1}\right)-r^{3}\left(s_{i}\right)=\sum_{j=1}^{\infty} \frac{(\Delta s)^{j}}{j !}\left(r^{3}\right)^{(j)}\left(s_{i}\right),
$$

and, therefore, the estimate becomes

$$
I_{2} \approx-\sum_{i=1}^{N} b_{2,2}^{\prime}\left(s_{i+1}\right) \sum_{j=1}^{\infty} \frac{(\Delta s)^{j}}{j !}\left(r^{3}\right)^{(j)}\left(s_{i}\right) .
$$

To estimate the behavior of $I_{2}$ as a function of aperture $D$, we first observe that the derivative $b_{2,2}^{\prime}(s)$ scales with $1 / D$, and thus

$$
b_{2,2}^{\prime}\left(s_{i+1}\right) \propto \frac{c_{i+1}}{D},
$$

where $c_{i+1}$ are suitable constants; similarly, we have

$$
\Delta s=s_{i+1}-s_{i}=\frac{s_{e}-s_{b}}{N} \propto \frac{D}{N} .
$$

Inserting (11) and (12) into (10) we obtain

$$
I_{2} \approx-\sum_{i=1}^{N} c_{i+1} \sum_{j=1}^{\infty} \frac{D^{j-1}}{j ! N^{j}}\left(r^{3}\right)^{(j)}\left(s_{i}\right) .
$$

In the sharp cutoff approximation, $D \rightarrow 0$, only the $j=1$ terms survive the limit. Thus, finally we obtain the qualitative behavior

$$
I_{2} \approx-\sum_{i=1}^{N} c_{i+1}^{*}\left(r^{3}\right)^{\prime}\left(s_{i}\right)
$$

where we absorbed various constants into $c_{i+1}^{*}$. Instead of being zero, the integral now approaches a constant and usually nonzero value as $D \rightarrow 0$. Therefore, the convergence to a constant of the second-order quadrupole tune shifts in the sharp cutoff limit can be qualitatively understood. We mention that the limit of the extension of fringe fields going to zero is equivalent to the limit of the apertures going to zero.

On the other hand, in the case of fourth-order tune shifts in the sharp cutoff limit, we obtain completely different

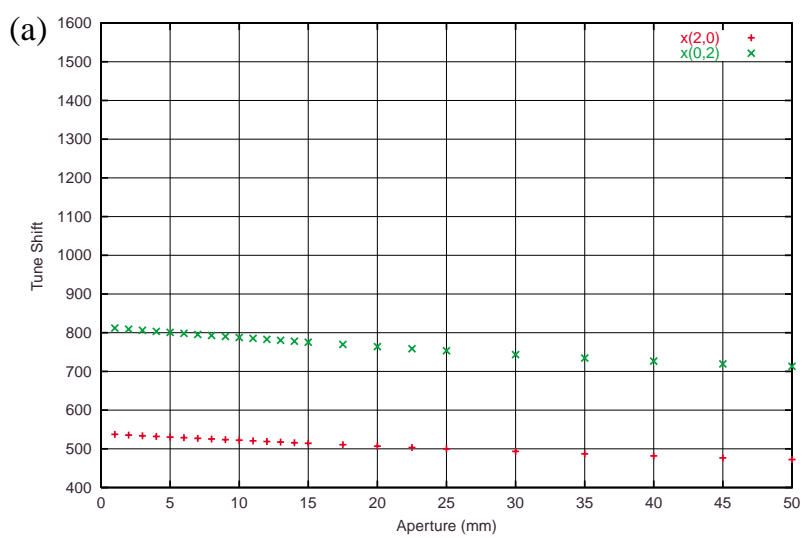

qualitative behavior. The fourth-order tune shifts are functions of fifth-order map elements. In this case, we need to estimate integrals of the type

$$
I_{4}=\int_{s_{b}}^{s_{e}} r^{5}(s) b_{2,2}^{(4)}(s) d s
$$

containing fourth-order derivatives of $b_{2,2}(s)$. Proceeding the same way as above we obtain that

$$
I_{4} \approx-\sum_{i=1}^{N} k_{i+1} \sum_{j=1}^{\infty} \frac{D^{j-3}}{j ! N^{j}}\left(r^{5}\right)^{(j)}\left(s_{i}\right),
$$

due to the fact that the third-order derivatives, $b_{2,2}^{(3)}\left(s_{i+1}\right)$, scale with $1 / D^{3}$. Hence, we obtain that in the sharp cutoff limit the integral diverges as

$$
I_{4} \approx \frac{a}{D^{2}}+\frac{b}{D}+c
$$

for some constants $a, b, c$. In a log-log plot the slope of the resulting curve will be between 0 and 2 depending on the exact values of $a, b$, and $c$. Generalizing this argument to a rectilinear $2 l$ pole, we see that the first divergence occurs always at order $2 l+1$.

The perturbative view employed in the above arguments can reveal the qualitative behavior of the situation, yet it is less fruitful for the quantitative study of the effects to very high orders of the motion, which would require the treatment of more and more integrals like those appearing above. In the map picture employed in COSY INFINITY, all dynamics can be solved by the ability of the differential algebraic (DA) approach to obtain the map to any order of interest, and then to obtain the tune shifts of interest to any order using DA-based normal form methods.

For the example of the default Cosy falloff, we explicitly obtained the divergence of the second-order and fourthorder amplitude dependent tune shifts; see Figs. 7 and 8. Only one second-order tune shift blows up, which shows that the single map element responsible for this effect comes from the dipole's $(b \mid$ yyy). This behavior can be seen in the logarithmic scale plots of Fig. 9, where slope 1 seems to be the limiting maximum slope. Although the

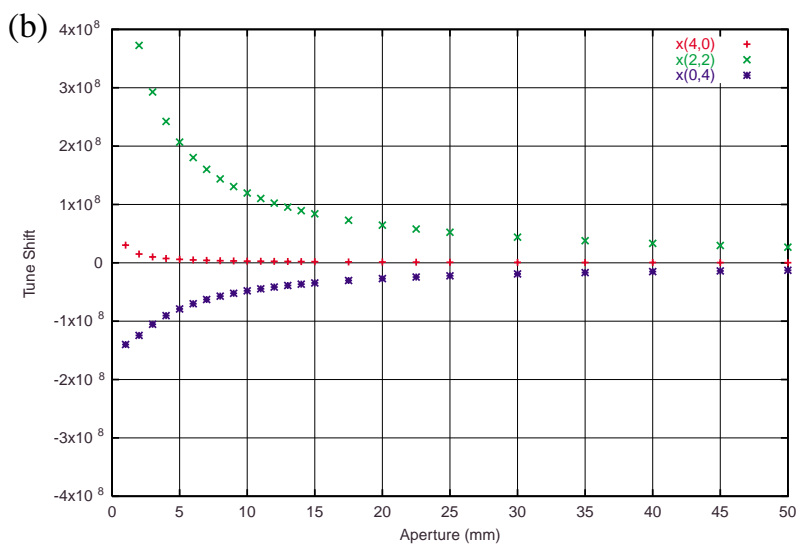

FIG. 7. (Color) (a) Convergence to a constant of the second-order and (b) blowup of the fourth-order amplitude dependent tune shifts in the $x$ tune. The fringe field shape is given by the default Enge coefficients. 

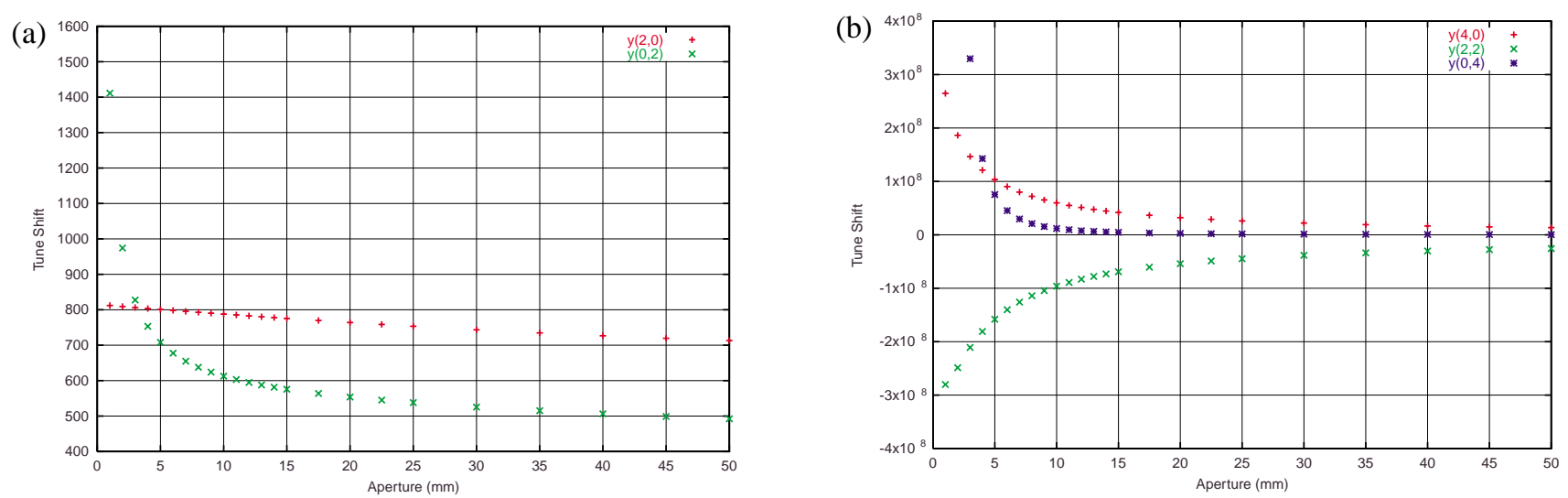

FIG. 8. (Color) (a) Blowup of the second-order and (b) fourth-order amplitude dependent tune shifts in the $y$ tune. The fringe field shape is given by the default Enge coefficients.
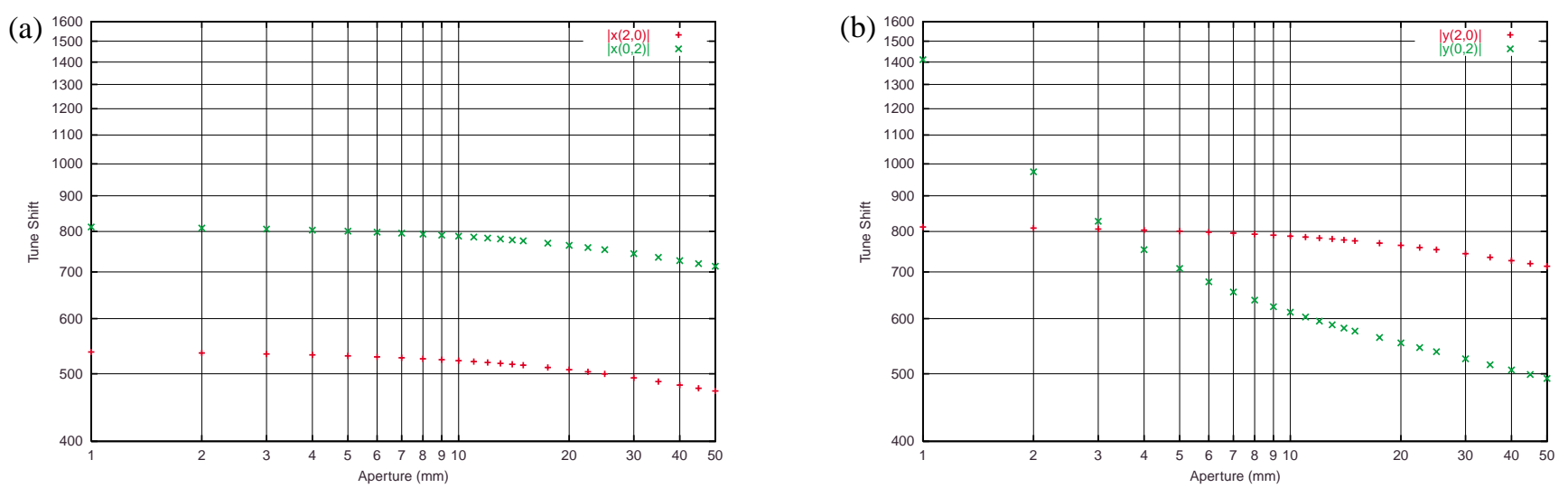

FIG. 9. (Color) Second-order amplitude dependent tune shifts as a function of aperture in $\log$-log scale: (a) $x$ tune shifts and (b) $y$ tune shifts. The maximum slope is around 1.
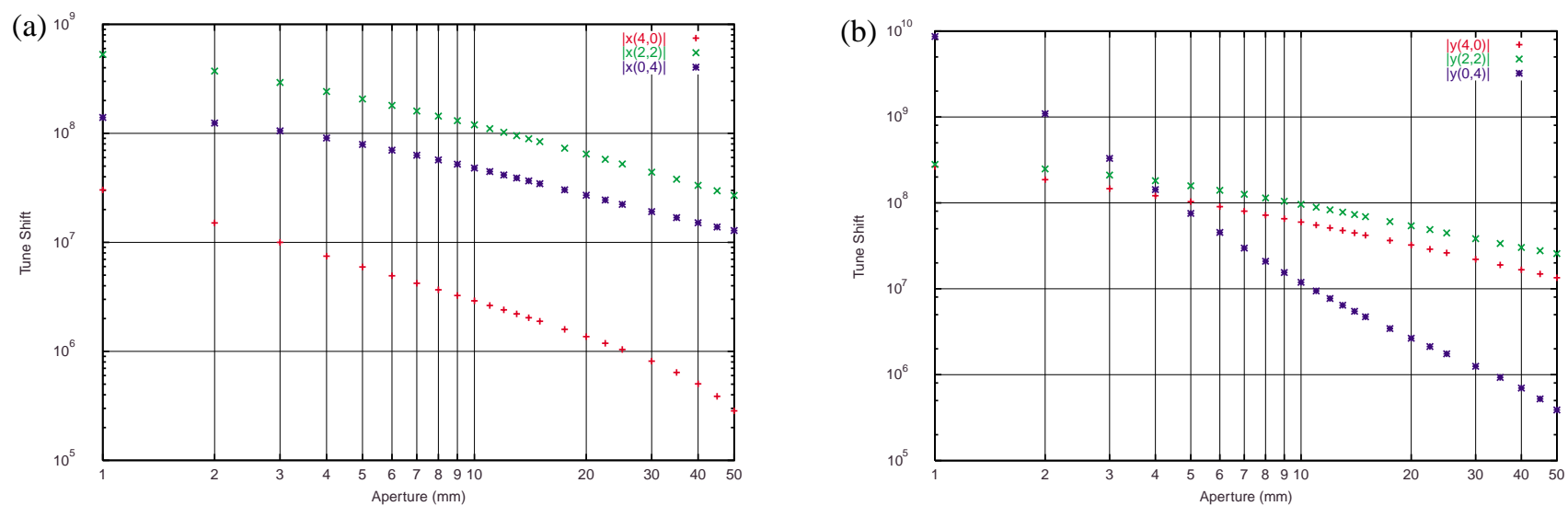

FIG. 10. (Color) Fourth-order amplitude dependent tune shifts as a function of aperture in $\log$-log scale: (a) $x$ tune shifts and (b) $y$ tune shifts. The maximum slope is around 3 .

constants $k_{i}(D)$ in general will be different at every time step, we expect that the divergence of some of the fifthorder quadrupole map elements will be roughly with the second power of the aperture. As it turns out, this also implies the divergence of the amplitude dependent tune shifts, and the logarithmic plots reveal the blowup with slope at most 3 (Fig. 10).

We performed the same studies for the ring with the matching quadrupole's fringe fields turned off. As expected, qualitatively we obtained the same results. The 
main difference is that the blowup of the fourth-order tune shifts begins at somewhat smaller apertures.

\section{SHAPE DEPENDENT EFFECTS}

Rescaling of the length of the fringe field region, for example, by changing the aperture, is a first example of shape dependent effect. This was studied in Sec. III. In this section we are interested in fall-off shape alterations, modulo translation, and rescaling. It can be achieved by different sets of Enge coefficients for the same lattice and different falloffs only for the quadrupoles. Also, the same falloff is assumed at both ends.

For example, one may want to choose a benign Enge function. This can be achieved by utilizing only the first two coefficients instead of six. Furthermore, one may want the same effective field boundary in both cases. Hence, for the Enge coefficients of the default case, we obtain the values listed in Table II. In the following we list two other sets, taken from fitting measured or simulated magnetic field data of specific quadrupoles. The Large Hadron Collider's high gradient quadrupoles of the interaction regions have been designed by Sabbi of Fermilab. Based on the magnet end design described in [30], we obtained the Enge coefficients listed in Table III [25]. Finally, another set has been obtained by Méot [24] for a warm, large aperture (diameter $\sim 30 \mathrm{~cm}$ ) quadrupole that belongs to a QD kaon spectrometer in operation at GSI; the values are $a_{1}=0.1122, a_{2}=6.2671, a_{3}=-1.4982, a_{4}=3.5882$, $a_{5}=-2.1209$, and $a_{6}=1.723$. These fits represent the fields globally, as well as along the optical axis.

Altogether, there is a total of six cases according to the above sets: quadrupoles with default dipole, default quadrupole, default sextupole, LHCHGQ lead end, two parameter default quadrupole Enge functions, and GSI QD spectrometer-type fringe fields. For each case we computed the maps at four different apertures: 25, 50, 75, and $100 \mathrm{~mm}$. By using the map, we obtained the tune shifts and resonance strengths via normal form methods [15], and the dynamic apertures by symplectic tracking with the order 8 map. For the tracking we followed the prescription of the optimal generating function symplectification (EXPO), described in [31,32].

Table IV represents the results of the computation of some of the amplitude dependent tune shifts in the hori-

TABLE II. Enge functions with only two parameters, computed by slightly modifying the second default Enge coefficient $\left(a_{2}\right)$ such that the corresponding Enge functions have the same integral.

\begin{tabular}{lrcr}
\hline \hline & Dipole & Quadrupole & Sextupole \\
\hline$a_{1}$ & -0.003183 & 0.00004 & -0.000117 \\
$a_{2}$ & 1.911302 & 4.518219 & 7.135786 \\
\hline \hline
\end{tabular}

zontal plane in all of the six cases for an aperture of $25 \mathrm{~mm}$. The same data is given in Tables V, VI, and VII for apertures of 50,75, and $100 \mathrm{~mm}$. Interestingly, there are only moderate changes, with both aperture and shape, of the second-order tune shifts, with the exception of a few cases where significant changes can be observed. For example, the LHCHGQ-type fringe fields, and to a lesser extent the default dipole-type, differ significantly from the other types when the aperture is around $75 \mathrm{~mm}$. Also, the small tune shift with horizontal action of the LHCHGQtype for aperture $25 \mathrm{~mm}$ is somewhat surprising. On the other hand, starting with the fourth order, the tune shifts depend significantly on the details of the fringe field shape.

In general, the results on the dynamic aperture and resonance strengths point in the same direction. Here, we include only some of the representative cases. We will present the results for the $75 \mathrm{~mm}$ aperture for all of the six fringe field shapes. The tracking pictures show the horizontal phase plane of on-energy particles launched along the $x$ axis with vanishing transversal momenta. The resonance strengths have been calculated along the diagonal in action space, at a value that approximately corresponds to the dynamic aperture.

We grouped the dynamic aperture pictures in Fig. 11 and the resonance strength pictures in Fig. 12 for the $75 \mathrm{~mm}$ aperture case. Notice that there is no real good correlation between the three different quantities computed. The lattice with dipole-type fringe field has larger than average amplitude dependent tune shifts and resonance strengths, which results in a smaller dynamic aperture. On the other hand, the LHCHGQ-type fringe fields result in even larger tune shifts, but the tracking shows a relatively clean looking phase space with an average dynamic aperture. Furthermore, in spite of the quadrupole-type fringe fields having larger resonance strengths than the sextupole-type, the dynamic apertures and the second-order tune shifts are approximately equal. Even between the maximum value of the resonance strengths of the six parameter, respectively the two parameter default quadrupole-type Enge function, there is a factor 5 difference in the resonance strengths, but they produce similar dynamic apertures. This may serve as an indication that it is wise to study fringe field effects on a case-by-case basis.

TABLE III. Enge coefficients fitted for the LHCHCQ lead and return ends, respectively.

\begin{tabular}{lrr}
\hline \hline & \multicolumn{1}{c}{ Lead end } & Return end \\
\hline$a_{1}$ & -0.939436 & -0.585368 \\
$a_{2}$ & 3.824163 & 3.603682 \\
$a_{3}$ & 3.882214 & 2.037629 \\
$a_{4}$ & 1.776737 & 0.768748 \\
$a_{5}$ & 0.296383 & 0.216590 \\
$a_{6}$ & 0.013670 & 0.035435 \\
\hline \hline
\end{tabular}


TABLE IV. A few amplitude dependent tune shifts for the $25 \mathrm{~mm}$ aperture case. All six of the studied fringe field fall-off shapes are included.

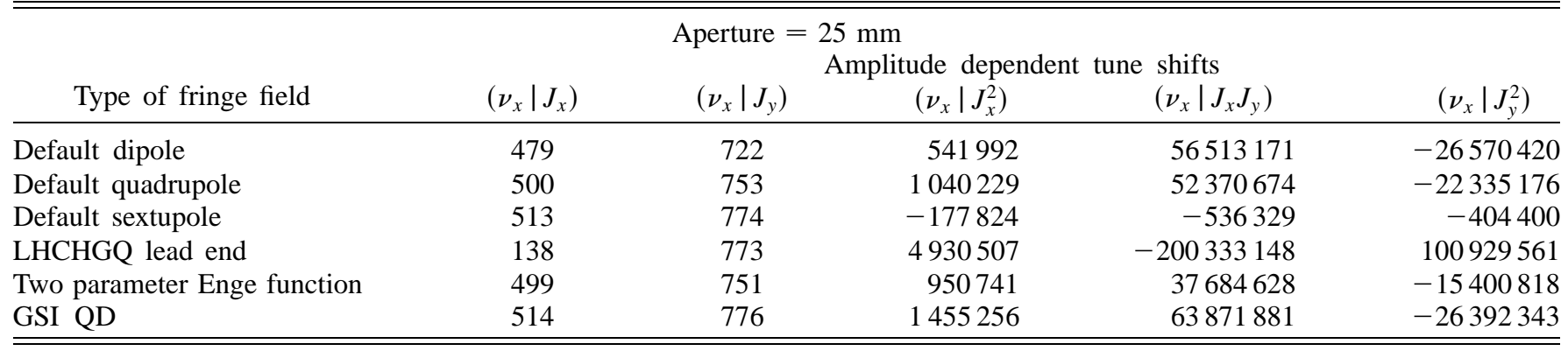

TABLE V. A few amplitude dependent tune shifts for the $50 \mathrm{~mm}$ aperture case. All six of the studied fringe field fall-off shapes are included.

\begin{tabular}{lccrr}
\hline \hline \multicolumn{1}{c}{ Aperture $=50 \mathrm{~mm}$} \\
Type of fringe field & $\left(\nu_{x} \mid J_{x}\right)$ & $\left(\nu_{x} \mid J_{y}\right)$ & $\begin{array}{c}\text { Amplitude dependent tune shifts } \\
\left(\nu_{x} \mid J_{x}^{2}\right)\end{array}$ & $\left(\nu_{x} \mid J_{x} J_{y}\right)$ \\
\hline Default dipole & 480 & 738 & -424782 & 44639316 \\
Default quadrupole & 472 & 713 & 284406 & 26926490 \\
Default sextupole & 492 & 741 & 727021 & -2591083 \\
LHCHGQ lead end & 411 & 953 & -19501 & -65824924 \\
Two parameter Enge function & 469 & 707 & 246449 & 1828937 \\
GSI QD & 494 & 746 & 522581 & 32686302 \\
\hline \hline
\end{tabular}

TABLE VI. A few amplitude dependent tune shifts for the $75 \mathrm{~mm}$ aperture case. All six of the studied fringe field fall-off shapes are included.

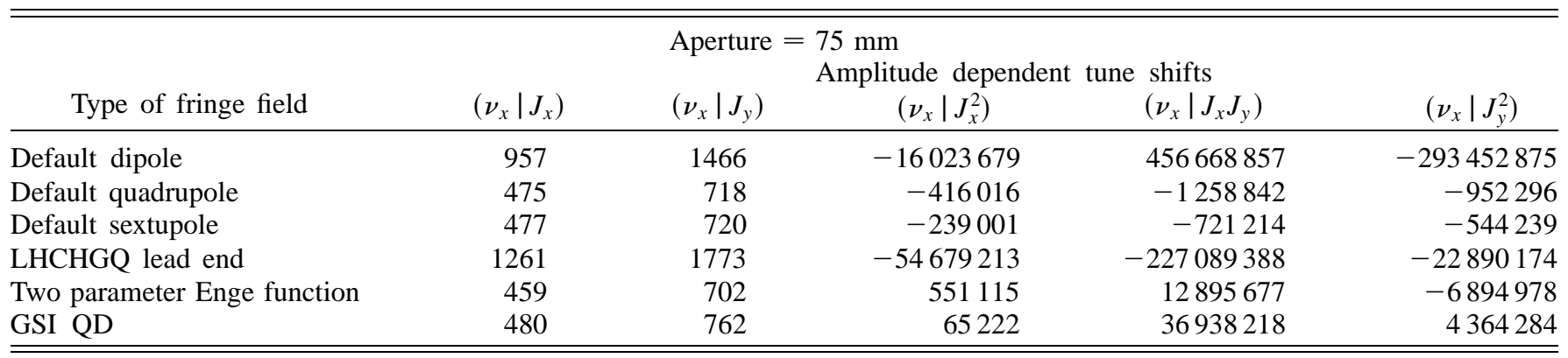

TABLE VII. A few amplitude dependent tune shifts for the $100 \mathrm{~mm}$ aperture case. All six of the studied fringe field fall-off shapes are included.

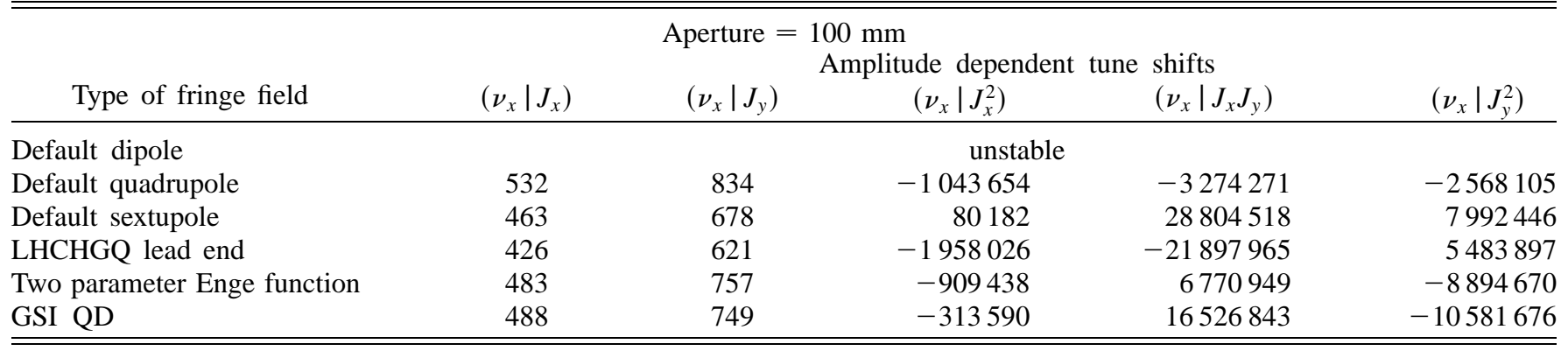

\section{SUMMARY AND CONCLUSIONS}

Recognizing that fringe fields might be important for the design of some of the proposed machines and accelerators under construction, we undertook a systematic study of the effects that one could expect. Using the example of the proposed $30 \mathrm{GeV}$ Neutrino Factory, we experimented with six different types of fringe fields at many different aperture settings. The main message of this paper is that fringe fields induce a variety of effects, and it is not always 

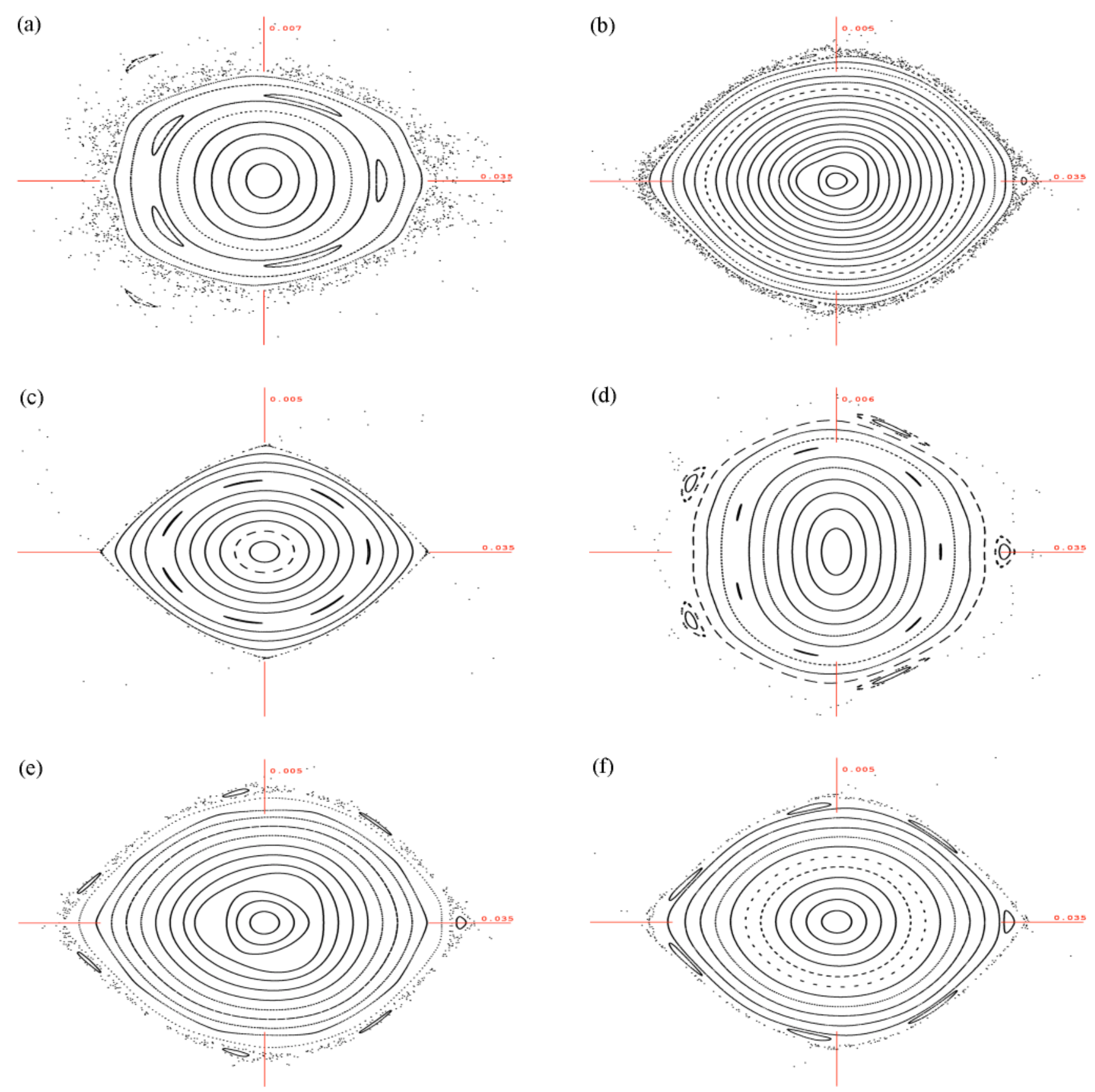

FIG. 11. (Color) Tracking pictures of on-energy particles launched along the $x$ axis with vanishing transversal momenta, magnet aperture of $75 \mathrm{~mm}$, for all six types of fringe fields. The following fringe field types are depicted: (a) default dipole, (b) default quadrupole, (c) default sextupole, (d) LHCHGQ lead end, (e) two parameter Enge function, and (f) GSI QD spectrograph.

straightforward to anticipate their effects without accurate simulation studies. The results point out that it is important to decide ahead of time on the end field designs for the proposed machines. We used fringe fields modeled by Enge functions for the Neutrino Factory because, at this stage, the exact shape is not known, as no model yet exists. As we mentioned, there is such a model for the LHCHGQs. Using differential algebraic techniques, it is possible to compute the multipole decomposition and accurate fringe field maps up to arbitrary order for such a model [21]. Once the necessary maps are available, the subsequent dynamical studies can proceed with no additional overhead compared to the case when no fringe field effects are taken into account. This includes normal form based quantities such as tune shifts, resonance strengths and resonance webs, and tracking. It is also worthwhile to note that symplectic tracking with fringe fields is of the same level of effort as without fringe fields [32].

Based on the magnet models described in [30], we performed a detailed study for the LHC [33] and found that 

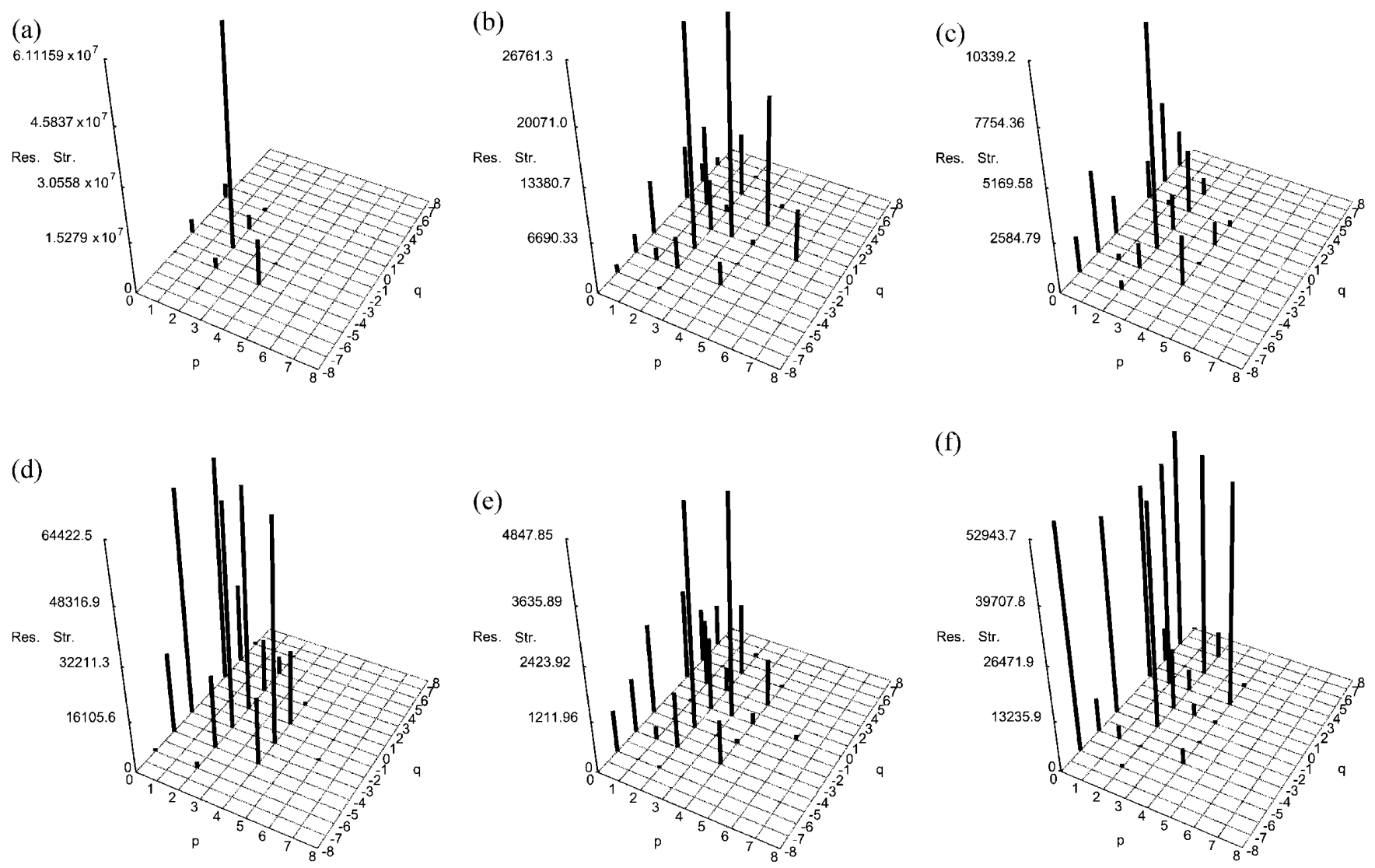

FIG. 12. Resonance strengths of on-energy particles along the diagonal in action space, at a distance from the origin that corresponds to the approximate dynamic aperture, magnet aperture of $75 \mathrm{~mm}$, for all six types of fringe fields. The following fringe field types are depicted: (a) default dipole, (b) default quadrupole, (c) default sextupole, (d) LHCHGQ lead end, (e) two parameter Enge function, and (f) GSI QD spectrograph.

the fringe field effects are noticeable, but not a limiting factor. We intend to undertake similar studies for the Neutrino Factory and the muon collider once the magnet end designs become available. In conclusion, a study of fringe field effects appears to be indicated for a detailed analysis of any ring lattice.

\section{ACKNOWLEDGMENTS}

We would like to thank C. Johnstone, F. Zimmermann, W. Wan, N. Holtkamp, J. Holt, M. Lindemann, G. Sabbi, M. Zisman, and E. Keil for useful discussions and collaborative efforts. This work was supported in part by the U.S. Department of Energy and an Alfred P. Sloan Foundation Fellowship.

[1] G. Lee-Whiting, Nucl. Instrum. Methods 83, 232 (1970).

[2] H. Matsuda and H. Wollnik, Nucl. Instrum. Methods 77, 40 (1970).

[3] H. Matsuda and H. Wollnik, Nucl. Instrum. Methods 77, 283 (1970).

[4] H. Matsuda and H. Wollnik, Nucl. Instrum. Methods 103, 117 (1972).
[5] B. Hartmann, H. Irnich, and H. Wollnik, in Nonlinear Problems in Accelerator Physics, edited by M. Berz, S. Martin, and K. Ziegler (Institute of Physics, Bristol, 1993), pp. 87-96.

[6] M. Venturini, in Proceedings of the 1999 Particle Accelerator Conference, New York (IEEE, New York, 1999).

[7] E. Forest and J. Milutinovic, Nucl. Instrum. Methods Phys. Res., Sect. A 269, 474 (1988).

[8] E. Forest et al., in Proceedings of the Fourth European Particle Accelerator Conference, London (World Scientific, London, 1994).

[9] H. Wollnik, Optics of Charged Particles (Academic, Orlando, 1987).

[10] M. Berz, K. Makino, and B. Erdelyi, in Colliders and Collider Physics at the Highest Energies, edited by B. J. King, AIP Conf. Proc. No. 530 (AIP, New York, 2000).

[11] F. Zimmermann et al., CERN Technical Reports No. SL2000-011 AP and No. NUFACT-NOTE 21, 2000 (unpublished).

[12] C. Johnstone, in Colliders and Collider Physics at the Highest Energies (Ref. [10]).

[13] M. Berz, Nucl. Instrum. Methods Phys. Res., Sect. A 298, 426 (1990).

[14] M. Berz, Part. Accel. 24, 109 (1989).

[15] M. Berz, Modern Map Methods in Particle Beam Physics (Academic, San Diego, 1999). 
[16] M. Berz, Los Alamos Report No. LA-11857-C, 1990, p. 137.

[17] M. Berz, Nucl. Instrum. Methods Phys. Res., Sect. A 298, 473 (1990).

[18] K. Makino and M. Berz, Nucl. Instrum. Methods Phys. Res., Sect. A 427, 338 (1999).

[19] M. Berz et al., The COSY INFINITY Web page, http: //www.beamtheory.nscl.msu.edu//cosy.

[20] M. Berz, National Superconducting Cyclotron Laboratory Report No. MSUCL-1088, 2000 (unpublished); see also http://www.beamtheory.nscl.msu.edu/cosy.

[21] B. Erdelyi, M. Lindemann, and M. Berz (to be published).

[22] K. L. Brown and J. E. Spencer, IEEE Trans. Nucl. Sci. 28, 2568 (1981).

[23] D. Bazin (private communication).

[24] F. Meot (private communication).

[25] W. Wan et al., Nucl. Instrum. Methods Phys. Res., Sect. A
427, 74 (1999).

[26] R. Abraham, J. E. Marsden, and T. Ratiu, Manifolds, Tensor Analysis, and Applications (Springer-Verlag, New York, 1988).

[27] I. Daubechies, Ten Lectures on Wavelets (SIAM, Philadelphia, 1992).

[28] L. Sagalovsky, Nucl. Instrum. Methods Phys. Res., Sect. A 298, 205 (1990).

[29] F. Zimmermann, CERN Technical Reports No. SL-2000009 AP and No. NUFACT-NOTE 18, 2000 (unpublished).

[30] G. Sabbi, Fermilab Technical Report No. TD-97-040, 1997 (unpublished).

[31] B. Erdelyi and M. Berz (to be published).

[32] B. Erdelyi, Ph.D. thesis (to be published).

[33] B. Erdelyi, M. Berz, and K. Makino, National Superconducting Cyclotron Laboratory Report No. MSUCL-1129, 1999 (unpublished). 